Introduction Patient satisfaction surveys (PSS) can help identify ways of improving practice and facilitate better quality care. Patient opinion in health services research is integral but data from chronic cough populations is unknown.

Aim To identify patient satisfaction in our tertiary cough service.

Methods We devised a PSS containing 19 structured questions. Patients attending review consultations in two consecutive clinics were asked to consider completing the anonymous PSS.

Results Fifty-two PSS were completed; an $84 \%$ response rate. Of those 43 had full responses for analysis [79\% female, $58 \% \geq 55$ years in age]. Patient satisfaction was extremely high (figure 1); 70\% thought the care received was excellent and $95 \%$ were likely to recommend the service to friends and family. Improvement suggestions related to parking and appointment management. However 44\% felt clinic locality was inconvenient, but the majority (63\%) of those were not interested in Skype review consultations; response was unrelated to age.

Conclusion To our knowledge, this is the first reported patient satisfaction data in chronic cough patients. Despite the refractory nature of the condition, patient satisfaction is extremely high. As a quarter of our service's patients travel $\geq 25$ miles, the inconvenience of clinic accessibility is not surprising. Nonetheless, patients appear to value face to face consultations and further patient consultation is required before utilising tele-health.

\section{M16 THEIR POINT OF VIEW: PATIENT EXPERIENCE OF A DGH PLEURAL SERVICE}

K Conroy, T Fretwell, H Tedd, J Killen. Queen Elizabeth Hospital, Gateshead, UK

\subsection{6/thoraxjnl-2017-210983.438}

Pleural disease constitutes a significant part of the workload on the respiratory unit but little information is recorded of the patient experience. We sought to record the experiences of our patients and look for ways to improve upon them.

Method We provided a brief questionnaire to in-patients and day attenders using our pleural service and recorded breathlessness, pain and anxiety on a visual analogue scale. A follow up questionnaire was collected within two weeks to record post procedure dyspnoea and time to improvement. We recorded pre-procedure questionnaires in 29 patients and 24 pre and post procedure in total from patients undergoing a mixture of procedures. Twelve had intercostal chest drains, eleven underwent therapeutic aspiration and six had diagnostic aspiration.

Results We found that patients undergoing intercostal drain insertion reported the greatest improvement in dyspnoea on visual analogue scale(65.8\% reduction), compared with $38 \%$ reduction in patients undergoing therapeutic aspiration. Excluding the diagnostic aspiration cohort, $85 \%$ of patients reported an improvement of their breathlessness over minutes or hours. Pain scores were similar between the therapeutic aspiration and chest drain group with $45 \%$ and $50 \%$ of patients reporting moderate to severe pain. We found the therapeutic aspiration group tended to report greater pre- procedure anxiety than the chest drain group. 63\% of patients in the therapeutic aspiration cohort reported moderate or severe pre-procedure anxiety, compared with only moderate or minor anxiety reported in the chest drain group. Fear of pain was particularly common in the therapeutic aspiration group. All patients reported feeling adequately prepared for the procedure. Despite this, when later asked how we could improve their experience four patients felt they would have liked more information about their procedure.

Conclusion Our study demonstrates that chest drain insertion is superior for dyspnoea relief and causes similar discomfort levels to therapeutic aspiration. Anxiety is common, but worse in the therapeutic aspiration group. A desire for more information on pleural procedures was identified.

Outcome We have introduced a patient information leaflet and have reviewed our anaesthetic and analgesia practice, and plan to repeat our questionnaire.

\section{M17 PATIENT \& CARER KNOWLEDGE OF PERSONALISED ASTHMA ACTION PLANS (PAAP)}

D Luciano, S Hails, P De Zwart, R Levey, S Moss. Great North Children Hospital, Newcastle Upon Tyne, UK

\subsection{6/thoraxjnl-2017-210983.439}

Introduction The National Review of Asthma Deaths (NRAD) and British Thoracic Society (BTS) guidance highlight the importance of patients with asthma having a PAAP. From ongoing local audit we know that over $90 \%$ of our clinic population are provided with a PAAP, which is updated on at least a yearly basis. However, we do not have data to inform us if the patients/carers use their plan appropriately.

Method A survey was conducted in a tertiary paediatric asthma clinic. The questionnaires were provided to parents and young adolescents with asthma whilst they were waiting for their appointment. There were specific yes/no and multiple choice questions as follows: location of paper plan, frequency of use, contacts and entities with access to plan, impression of unscheduled healthcare reduction and a direct question asking if digitalizing a personalised asthma plan would be useful.

Results 55 questionnaires were completed. All but 1 patient were able to identify that they had a PAAP. The majority of individuals considered their PAAP useful (90\%) and they all found it easy to follow. $67 \%$ of patients looked at their PAAP on at least a monthly basis. 19 patients had not shared their PAAPs with other carers, whilst PAAPs had been shared with schools (30), grandparents (13) and childminder (2). Patients/ carers perceived that knowledge of their PAAPs had helped to reduce unscheduled healthcare attendances (89\%) and symptoms (49\%); with $47 \%$ perceiving it had helped increase their peak expiratory flow rate (PEFR). 95\% of all patients would prefer a PAAP in an electronic format.

Conclusion In tertiary paediatric asthma clinic patients and their carers have good knowledge of their PAAP, use them regularly and share them with other care givers. They perceive that they do help improve asthma control and reduce exacerbations. Families would prefer their PAAP in an electronic format. 